University of New Hampshire

University of New Hampshire Scholars' Repository

Physics Scholarship

Physics

\title{
2-2015
}

\section{Dielectric breakdown weathering of the Moon's polar regolith}

Andrew P. Jordan

University of New Hampshire, A.P.Jordan@unh.edu

T. J. Stubbs

Goddard Space Flight Center

Jody K. Wilson

University of New Hampshire, jody.wilson@unh.edu

Nathan A. Schwadron

University of New Hampshire, Nathan.Schwadron@unh.edu

Harlan E. Spence

University of New Hampshire, harlan.spence@unh.edu

Follow this and additional works at: https://scholars.unh.edu/physics_facpub

Part of the Astrophysics and Astronomy Commons

\section{Recommended Citation}

A. P. Jordan, T. J. Stubbs, J. K. Wilson, N. A. Schwadron, and H. E. Spence, 'Dielectric breakdown weathering of the Moon's polar regolith', Journal of Geophysical Research: Planets, vol. 120, no. 2, pp. 210-225, Feb. 2015.

This Article is brought to you for free and open access by the Physics at University of New Hampshire Scholars' Repository. It has been accepted for inclusion in Physics Scholarship by an authorized administrator of University of New Hampshire Scholars' Repository. For more information, please contact Scholarly.Communication@unh.edu. 
Journal of Geophysical Research: Planets

\author{
RESEARCH ARTICLE \\ 10.1002/2014JE004710 \\ Key Points: \\ - Large solar energetic particle \\ events may cause breakdown in \\ lunar regolith \\ - We estimate the rate of these \\ breakdown-causing particle events \\ - Breakdown weathering may change \\ the properties of polar regolith
}

\section{Correspondence to:}

A. P. Jordan,

a.p.jordan@unh.edu

\section{Citation:}

Jordan, A. P., T. J. Stubbs, J. K. Wilson, N. A. Schwadron, and H. E. Spence (2015), Dielectric breakdown weathering of the Moon's polar regolith, $J$. Geophys. Res. Planets, 120, 210-225, doi:10.1002/2014JE004710.

\section{Received 6 AUG 2014}

Accepted 15 JAN 2015

Accepted article online 22 JAN 2015

Published online 19 FEB 2015

\section{Dielectric breakdown weathering of the Moon's polar regolith}

\author{
A. P. Jordan ${ }^{1,2}$, T. J. Stubbs ${ }^{2,3}$, J. K. Wilson ${ }^{1,2}$, N. A. Schwadron ${ }^{1,2}$, and H. E. Spence ${ }^{1,2}$ \\ ${ }^{1}$ Institute for the Study of Earth, Oceans, and Space, University of New Hampshire, Durham, New Hampshire, USA, \\ ${ }^{2}$ Solar System Exploration Research Virtual Institute, NASA Ames Research Center, Moffett Field, California, USA, \\ ${ }^{3}$ NASA Goddard Space Flight Center, Greenbelt, Maryland, USA
}

Abstract Galactic cosmic rays and solar energetic particles (SEPs) can charge the Moon's subsurface, a process expected to be particularly important in the polar regions. Experiments have shown that sufficient fluences (i.e., time-integrated fluxes) of energetic charged particles can cause dielectric breakdown, in which the electric field rapidly vaporizes small, filamentary channels within a dielectric. Lunar regolith has both the characteristics and, in some polar locations, the environment needed to make breakdown likely. We combine the Jet Propulsion Laboratory proton fluence model with temperature measurements from the Lunar Reconnaissance Orbiter's (LRO's) Diviner instrument and related temperature modeling to estimate how often breakdown occurs in the polar regions. We find that all gardened regolith within permanently shadowed regions (PSRs) has likely experienced up to $2 \times 10^{6}$ SEP events capable of causing breakdown, while the warmest polar regions have experienced about 2 orders of magnitude fewer events. We also use measurements from the Cosmic Ray Telescope for the Effects of Radiation on LRO to show that at least two breakdown-inducing events may have occurred since LRO arrived at the Moon in 2009. Finally, we discuss how such "breakdown weathering" may increase the percentage of fine and monomineralic grains within PSRs; explain the presence of so-called "fairy castle" regolith structures; and contribute to other low-albedo features detected by LRO's Lyman Alpha Mapping Project, possibly establishing a correlation between these features and the average temperatures within craters that are only partly in permanent shadow.

\section{Introduction}

The Moon can experience significant surface charging, as a number of studies have shown. One ongoing source of charging is the surface's direct exposure to solar radiation and the ambient space plasma environment, such as the solar wind [Stubbs et al., 2014]. The presence of a global-scale plasma wake downstream of the Moon significantly affects surface charging over most of the lunar nightside [Halekas et al., 2008]. Similarly, miniwakes, which form downstream of obstacles (e.g., crater rims) near the terminator, are predicted to control the access of plasma and surface charging in permanently shadowed regions (PSRs) [Farrell et al., 2010; Zimmerman et al., 2011, 2012]. Also, large solar energetic particle (SEP) events can cause the surface potential on the lunar nightside to reach a few kilovolts negative [Halekas et al., 2007, 2009].

None of these studies, however, considered the effects of deep dielectric charging in the lunar subsurface. Jordan et al. [2014] were the first to show how galactic cosmic rays and SEPs can charge the polar subsurface, particularly within PSRs, where the electrical conductivity is predicted to be very low. By creating a data-driven, one-dimensional, time-dependent model, they found that large SEP events may even cause dielectric breakdown, in which the electric field $\left(\geq 10^{7} \mathrm{~V} / \mathrm{m}\right)$ quickly vaporizes small channels within regolith grains. They estimated that all gardened regolith within PSRs has experienced about $10^{6}$ SEP events capable of causing breakdown.

In this paper, we consider this process in more detail. We describe some of the laboratory work done to understand breakdown in various materials, including rocks. We combine the Jet Propulsion Laboratory (JPL) proton fluence model with temperature measurements from the Lunar Reconnaissance Orbiter's (LRO's) Diviner instrument and related modeling to estimate an occurrence rate of SEP events capable of causing dielectric breakdown, which can be applied to the Moon's polar regions. This rate enables us to assess how breakdown may affect the lunar regolith in different locations. We also demonstrate how to 
use the Cosmic Ray Telescope for the Effects of Radiation (CRaTER) on the Lunar Reconnaissance Orbiter to identify new potentially breakdown-inducing SEP events.

\section{Breakdown and the Electrical Properties of Lunar Regolith}

Dielectric breakdown occurs if the electric field within a material exceeds a threshold which depends on its composition and geometry. If a breakdown electric field is created by the deposition of energetic charged particles, then they must have been deposited within the material's characteristic discharging timescale $\tau$, where

$$
\tau=\epsilon / \sigma_{c}
$$

and $\epsilon$ is its permittivity and $\sigma_{c}$ its conductivity (cf. the derivation by Buhler et al. [2007]). The required fluence (time-integrated flux) of charged particles is $\sim 10^{10}-10^{11} \mathrm{~cm}^{-2}$ for breakdown in solids [Frederickson et al., 1992; Violet and Frederickson, 1993; Garrett and Evans, 2001; Green and Dennison, 2008]. If these criteria are met, the dielectric atomizes and is converted to a plasma within small, filamentary, tree-like channels [Budenstein, 1980], which can be about $10 \mu \mathrm{m}$ in diameter [Budenstein et al., 1969]. As summarized by Budenstein [1980] and Frederickson et al. [1986], breakdown within most solids typically occurs at $\sim 10^{7} \mathrm{~V} / \mathrm{m}$. Some laboratory experiments show that inhomogeneities within dielectrics can cause breakdown to occur with electric fields as low as $10^{6} \mathrm{~V} / \mathrm{m}$ [Sørensen et al., 1999]. For a more detailed review, see the discussion in Jordan et al. [2014].

Therefore, given a sufficient flux and fluence of SEPs, breakdown may occur in the lunar regolith. We next show that it is indeed conducive to breakdown, especially at certain locations in the polar regions. Then, to find the SEP conditions needed for breakdown, we describe the regolith's electrical characteristics.

Lunar regolith is conducive to breakdown for several reasons. First, its grains tend to have irregular and jagged shapes. These cusp-like projections can increase local electric fields by $1-2$ orders of magnitude with respect to the average electric field [Bahder et al., 1982].

Second, inclusions are frequent within much of the regolith's grains, particularly the larger ones. Three of the five basic regolith particles_lithic clasts, breccias, and agglutinates-by their nature contain multiple dielectrics [McKay et al., 1991], while even the regolith's glassy component sometimes contains inclusions [McKay et al., 1991]. The boundaries between these different dielectrics increases the local electric field, thus decreasing the material's resistance to dielectric breakdown [Hara and Okubo, 1998; Lisitsyn et al., 1998; Fujita et al., 2001; Andres et al., 2001a, 2001b].

Third, regolith grains also sometimes have gas inclusions; Roedder and Weiblen [1970] discovered that glass inclusions $>3 \mu \mathrm{m}$ diameter contain bubbles of gas and vacuum, likely formed as the glass and surrounding crystal shrank differently during cooling. They also found gas inclusions outlining healed fractures within olivine crystals. Because vacuum inclusions cannot form in growing olivine, the inclusions must be gas filled. Funkhouser et al. [1971] also found gas-filled vesicles in rocks-more commonly breccias than crystalline rocks. Such gas-filled cavities may aid in the destruction of grains during breakdown, since they have a lower dielectric strength than the solid. The ionization of the gas causes a microexplosion, stressing the rock, and repeating these stresses eventually destroys the rock [Lisitsyn et al., 1998].

To our knowledge, only one series of experiments has studied breakdown in regolith [Kirkici et al., 1996]. The experimenters placed electrodes separated by $1 \mathrm{~mm}$ in a vacuum container with regolith simulant (Minnesota lunar simulant \#1) at room temperature. They found that the simulant (conductivity $\approx 10^{-14} \mathrm{~S} / \mathrm{m}$ ) experienced breakdown at electric fields of $6 \mathrm{MV} / \mathrm{m}$. They did not detect any optical emission, nor could they find, through microscopic observation, any physical damage to the regolith. The lack of damage was unexpected, since breakdown necessitates some weathering. Regardless, the study does indicate that breakdown may occur in the regolith. (Note that the simulant they used was less "jagged" than typical regolith, so true regolith may be even more conducive to breakdown than the simulant.)

To find the SEP conditions needed for breakdown, we must first estimate the regolith's discharging timescale $\tau$, which, in turn, depends on both its permittivity $\epsilon$ and its conductivity $\sigma_{c}$. Olhoeft and Strangway [1975] found that the regolith's dielectric constant (i.e., relative permittivity) is typically $\approx 2$ and likely constant over the temperature range we consider here [Olhoeft et al., 1974a]. Therefore, we assume the lunar regolith's permittivity to be $\epsilon=2 \epsilon_{0}$. 


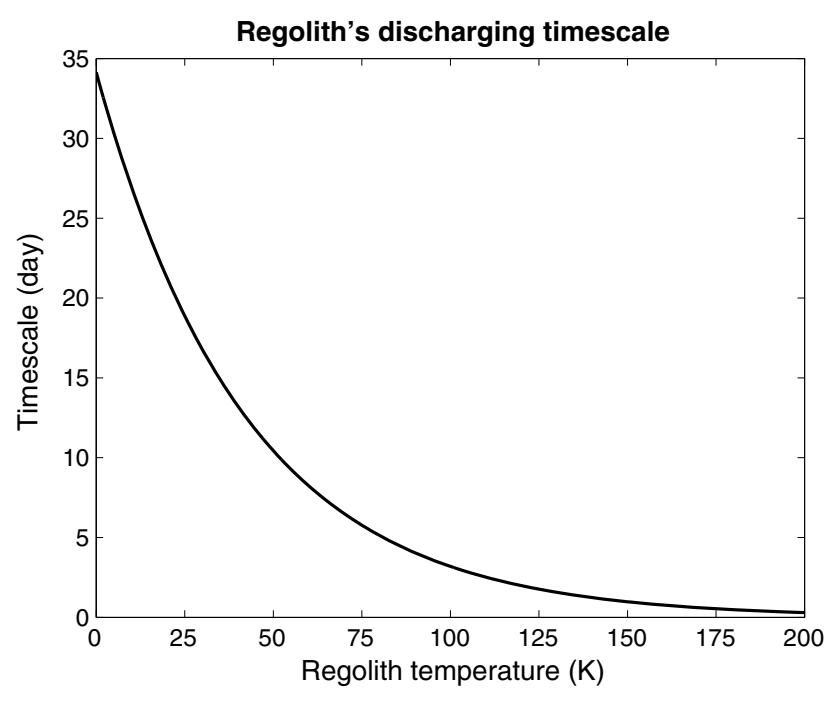

Figure 1. The characteristic electrical discharging timescale of the lunar regolith as a function of temperature.
The regolith's electrical conductivity is temperature dependent. An Apollo 15 soil sample's conductivity (in units of siemens per meter) was

$$
\sigma_{c}=\sigma_{c 0} e^{\alpha T}
$$

where $\sigma_{c 0}=6 \times 10^{-18} \mathrm{~S} / \mathrm{m}$, $\alpha=0.0237 \mathrm{~K}^{-1}$, and $T$ is the temperature in kelvin [Olhoeft et al., 1974b]. This relationship may change depending on the mineralogical characteristics of the regolith at any given location, but we assume it adequately describes the entire polar region. Also, although this relationship was determined for temperatures between 300 and $1100 \mathrm{~K}$-higher than the exceptionally cold temperatures typical of PSRs-it is, to the best of knowledge, the best characterization currently available.

This temperature dependence means that regolith at the colder PSR temperatures (tens of kelvins) [Paige et al., 2010] dissipates internal charge more slowly than warmer regolith (such as that used in the above experiments by Kirkici et al. [1996]), because its electrical conductivity is much lower. This slower dissipation therefore increases the magnitude of the subsurface electric field that can form [Jordan et al., 2014]. Figure 1 shows the discharging timescale's dependence on temperature. Above $160 \mathrm{~K}$, the timescale is so short that the possibility of breakdown can be neglected. Within PSRs, discharging can take $\sim 20$ days, which is a significant fraction of a lunation.

We use the modeling results of Paige et al. [2010] to estimate both the typical and the minimum discharging timescales at both poles (see Figure 2). The authors fit a thermal model to the Diviner north and south polar observations to estimate the average annual temperature at a depth of $2 \mathrm{~cm}$ (see their Figure 1c) and the maximum surface temperature. We note that the model's uncertainties, $<7 \mathrm{~K}$ in the warmest craters but near zero in the coldest, have only a limited effect on our calculations. Also, although the range of temperatures at $1 \mathrm{~mm}$ (the typical penetration depths of SEPs [Jordan et al., 2014]) is greater than the range of temperatures at $2 \mathrm{~cm}$, the thermal gradient in the lunar subsurface is such that the average temperature at $1 \mathrm{~mm}$ is actually colder than the average temperature at $2 \mathrm{~cm}$. The temperature difference between depths of $1 \mathrm{~mm}$ and $2 \mathrm{~cm}$ is likely only a few kelvins [Paige et al., 2010].

The maps in Figure 3 (left column) show the average discharging timescale, assuming the temperatures at $1 \mathrm{~mm}$ are equal to those at $2 \mathrm{~cm}$ depth. The longest discharging timescales are 10-20 days. Similarly, the maps in Figure 3 (right column) show the minimum discharging timescale, using the maximum surface temperature. Even at these high temperatures, PSRs still typically have discharging timescales of about 5 days.

Despite the presence of water ice within and on the surface of some PSRs [Colaprete et al., 2010; Gladstone et al., 2012], the regolith's overall conductivity likely remains unaffected. [Colaprete et al., 2010] stated that at least some of the water (5.6 $\pm 2.9 \%$ by mass) released in the Lunar Crater Observation and Sensing Satellite (LCROSS) impact in Cabeus crater was in the form of ice. Neish et al. [2011], using Mini-RF on LRO and Mini-SAR on Chandrayaan-1, found that the water in Cabeus must be either in $\lesssim 10 \mathrm{~cm}$ ice fragments or as a hydroxyl compound adsorbed on the regolith grains. Gladstone et al. [2012] reported that some PSRs (excluding Cabeus) contain 1-2\% surface water frost by area. Since ice grains comprise such a small fraction of the regolith, they are unlikely to form enough conduction channels to affect the regolith's conductivity. Furthermore, as indicated by laboratory studies of pure ice (see the review by Petrenko [1993]), such low temperatures likely give the ice a conductivity that may be orders of magnitude lower than that of the regolith. 

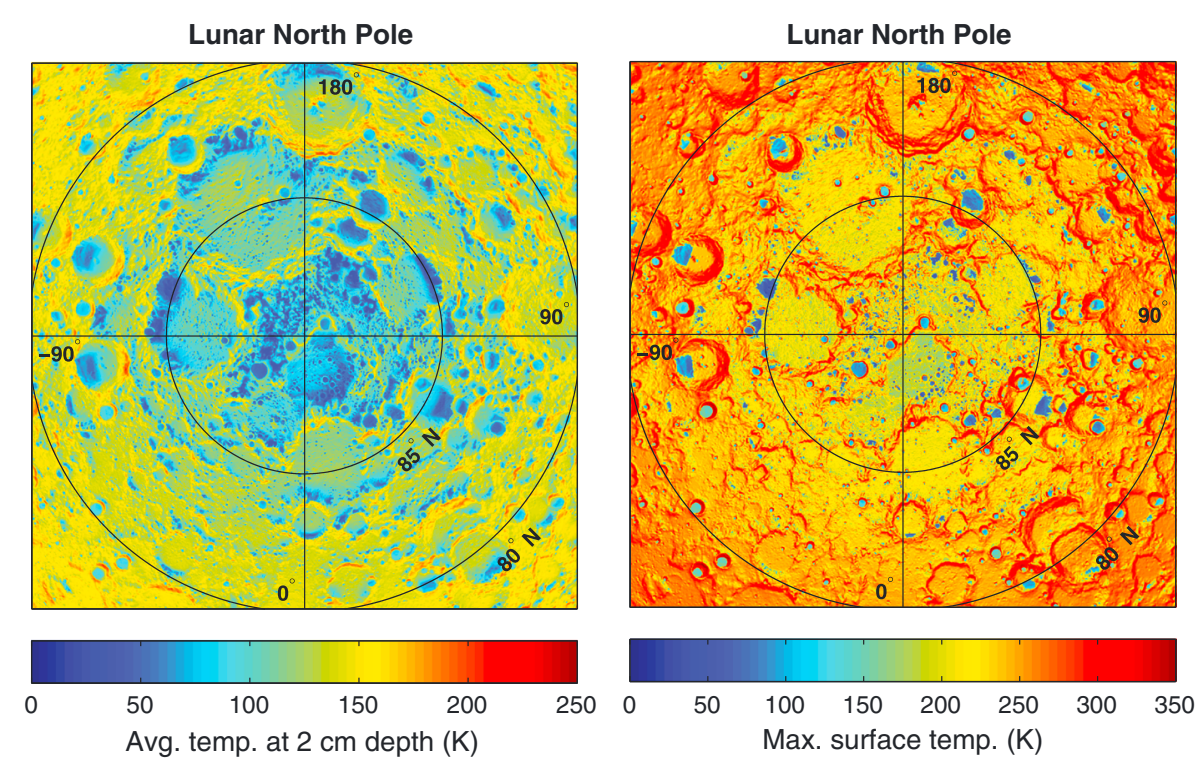

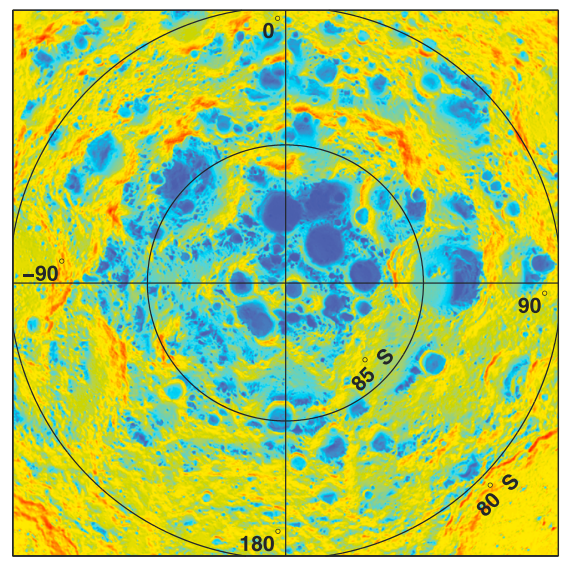

Lunar South Pole

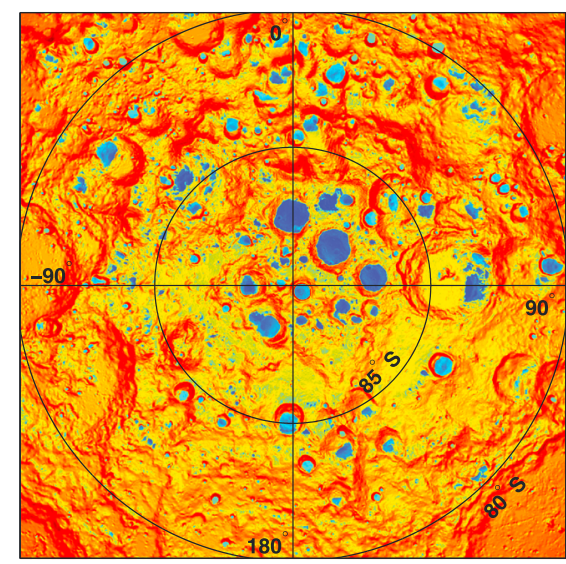

Lunar South Pole

Figure 2. (left column) The annual average temperature at $2 \mathrm{~cm}$ depth and (right column) the annual maximum surface temperature. Note that the two temperature scales are not the same.

Lunar regolith therefore has both the characteristics and, in some polar locations, the environment needed to make breakdown likely. As shown by Jordan et al. [2014], SEP events that are predicted to be capable of causing breakdown occur, on average, about once per year. Building on that study, we investigate how the conditions for breakdown depend on surface temperature, and thus conductivity, which enables us to identify the specific polar locations at which breakdown could occur.

\section{Frequency of Breakdown-Inducing Solar Energetic Particle Events}

Solar energetic particles are the primary means of charging the lunar subsurface. These SEPs are ions and electrons accelerated in solar flares and the shocks of coronal mass ejections to energies of $\sim 50 \mathrm{keV}-10 \mathrm{GeV}$ and $\sim 1 \mathrm{keV}-10 \mathrm{MeV}$, respectively, with the spectral peaks varying by event [Gosling, 1993; Reames, 1999; McGuire and von Rosenvinge, 1984]. SEP events are episodic and tend to occur more often near solar maximum than near minimum [Smart and Shea, 1985]. Work on lunar samples by Russ and Emerson [1980] and Reedy [1980] indicates that SEP fluxes over the past 10 Myr were similar to modern fluxes.

At the energies we consider, both SEP electrons and protons have gyroradii on the order of or greater than the Moon's radius, so we assume they are isotropic. Also, as mentioned in Jordan et al. [2014], the rims of large craters (diameters $>15 \mathrm{~km}$ ) block little or none of the sky, and the rims of smaller craters block only 

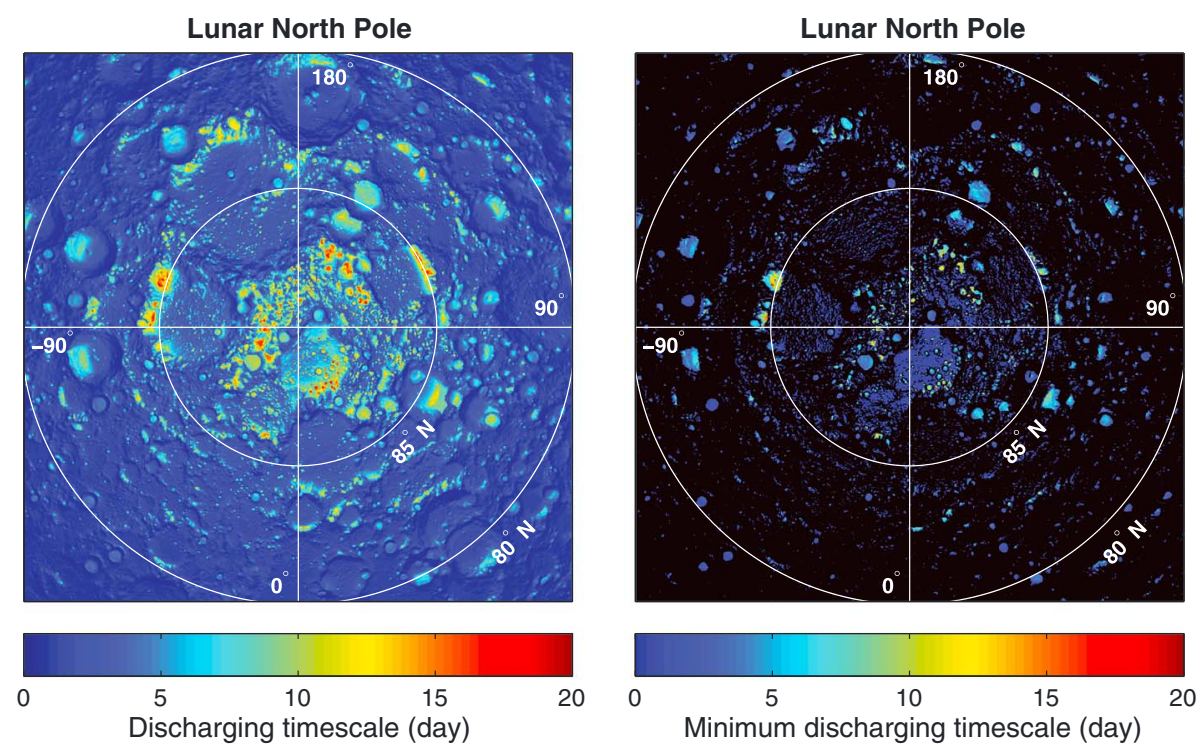

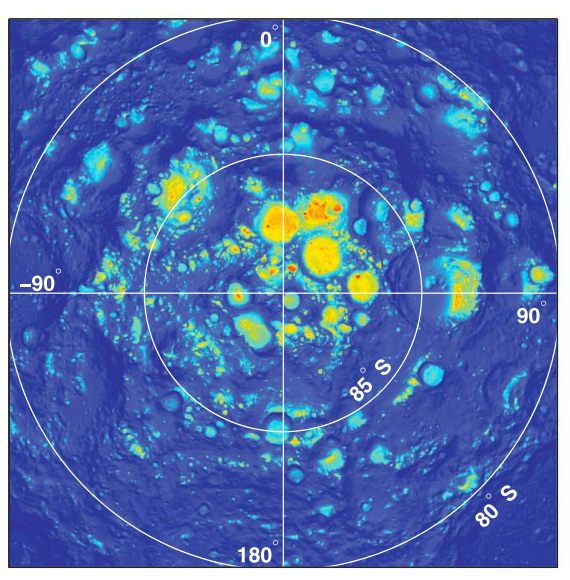

Lunar South Pole

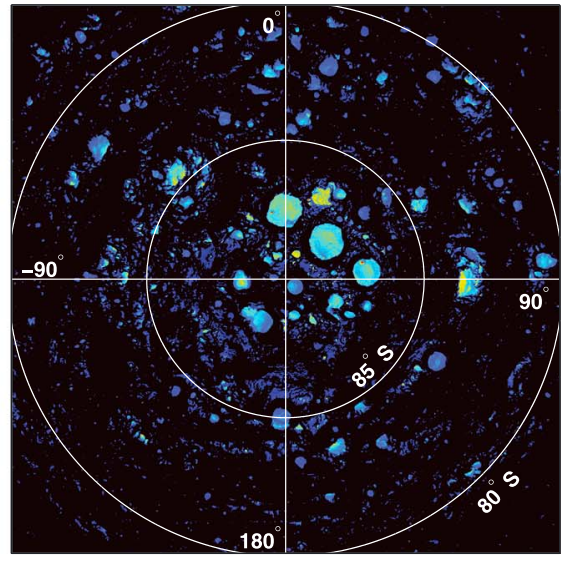

Lunar South Pole

Figure 3. Maps of the typical and minimum discharging timescales, derived from the temperature maps. (left column) Based on average temperature at $2 \mathrm{~cm}$ depth and (right column) derived assuming regolith remains at the annual maximum surface temperature. Black-colored regions are where the maximum temperature $>200 \mathrm{~K}$.

about a third. We therefore assume the lunar surface, including PSRs, is exposed to energetic particles emanating from space over a solid angle of $2 \pi \mathrm{sr}$.

For the purposes of estimating the frequency of breakdown-inducing SEP events, it is sufficient to consider only protons for this study, since peak proton fluxes are generally 2-3 orders of magnitude higher than peak electron fluxes [Evenson et al., 1984; Cane et al., 1986]. Since very few events have higher-peak electron fluxes, the resulting subsurface electric field is usually due mainly to the protons. We thus assume the proton fluence to be an adequate proxy for breakdown.

To estimate the rate of breakdown-inducing SEP events, we use the JPL proton fluence model [Feynman et al., 1990, 1993, 2002], which was developed to estimate the fluence of solar energetic protons that spacecraft would receive over their lifetimes. Feynman et al. [2002] have shown that the model, although last refined in 1993, correctly accounted for events that had occurred since then (by 2002, it accounted for a total of 35 years of data). During that period, the authors measured the proton fluence for various integral energies during the seven active years of each solar cycle: the 2 years preceding solar maximum, the year of maximum, and the ensuing 4 years. They found that the occurrence frequency of SEP event fluences for any integral energy channel fit a lognormal distribution. 
Feynman et al. [1993] identified SEP events using proton integral energy channels from the IMP and OGO spacecraft, the lowest channel of which were $>1 \mathrm{MeV}$. $1 \mathrm{MeV}$ protons penetrate to $\sim 20 \mu \mathrm{m}$, while most $10 \mathrm{MeV}$ protons penetrate to about $0.5 \mathrm{~mm}$ and some to about $1 \mathrm{~mm}$, depending on their incidence angle. We therefore assume that the entire $>1 \mathrm{MeV}$ channel penetrates to roughly $1 \mathrm{~mm}$ [Jordan et al., 2014]. Of the nearly 19 years available for this energy range, Feynman et al. [1993] found 89 large events during which the daily averaged flux exceeded $460 \mathrm{~cm}^{-2} \mathrm{~s}^{-1} \mathrm{sr}^{-1}$ ( $\sim$ events/yr). Even if some large events occurred during the four unanalyzed years of solar minimum, they would only slightly affect this rate. We therefore conservatively assume none occur during minimum. Feynman et al. [1993] found the mean $\mu$ of the lognormal distribution for these events' fluences to be $\log _{10}\left(3.0 \times 10^{9} \mathrm{~cm}^{-2}\right)$ with a standard deviation $\sigma$ of 0.61 .

This distribution enables us to estimate the occurrence rate for events that can cause breakdown in lunar regolith of a given temperature. These events must meet two criteria. First, they must deposit enough charged particles to create sufficiently strong subsurface electric fields (fluence). Second, they must deposit those particles faster than the regolith can dissipate them (flux). This dissipation, i.e., the discharging timescale $\tau$, depends on temperature. To find the frequency of events meeting these two criteria, we first estimate the rate of events meeting or exceeding a given fluence. Next, we find a characteristic event duration to estimate the average flux of any given event.

First, we define the proton fluence $f_{p}$ of an event to be

$$
f_{P}=10^{F} \mathrm{~cm}^{-2}
$$

As Feynman et al. [1990] show, the distribution of these event fluences is lognormal, i.e., Gaussian with respect to $F$, so the probability density function $p(F)$ of event fluence is

$$
p(F)=\frac{1}{\sqrt{2 \pi} \sigma} \exp \left[\frac{-(F-\mu)^{2}}{2 \sigma^{2}}\right]
$$

where $\mu$ is the mean and $\sigma$ is the standard deviation of the events (defined above). The fraction of events with fluences $\geq f_{p}$ (or the log of fluences $\geq F$ ) is therefore

$$
n(\geq F)=\int_{F}^{\infty} p\left(F^{\prime}\right) \mathrm{d} F^{\prime}=\frac{1}{\sqrt{2 \pi} \sigma} \int_{F}^{\infty} \exp \left[\frac{-\left(F^{\prime}-\mu\right)^{2}}{2 \sigma^{2}}\right] \mathrm{d} F^{\prime}
$$

To simplify this equation, we can set

$$
t=\frac{F-\mu}{\sqrt{2} \sigma}
$$

and substitute it into equation (5) to get

$$
n(\geq F)=\frac{1}{\sqrt{\pi}} \int_{\frac{F-\mu}{\sqrt{2} \sigma}}^{\infty} e^{-t^{2}} \mathrm{~d} t
$$

Since the complementary error function is

$$
\operatorname{erfc}(z)=\frac{2}{\sqrt{\pi}} \int_{z}^{\infty} e^{-t^{2}} d t
$$

it follows that

$$
n(\geq F)=\frac{1}{2} \operatorname{erfc}\left(\frac{F-\mu}{\sqrt{2} \sigma}\right)
$$

Again, this is the fraction of events with fluences greater than $10^{F} \mathrm{~cm}^{-2}$ (see Figure 4). As mentioned above, the rate of events in this integral energy channel $\left(>1 \mathrm{MeV}\right.$ ) is roughly $R_{0}=5$ events/yr. Therefore, the rate $R$ of events with fluences greater than $10^{F}$ is

$$
R(\geq F)=R_{0} n(\geq F)
$$




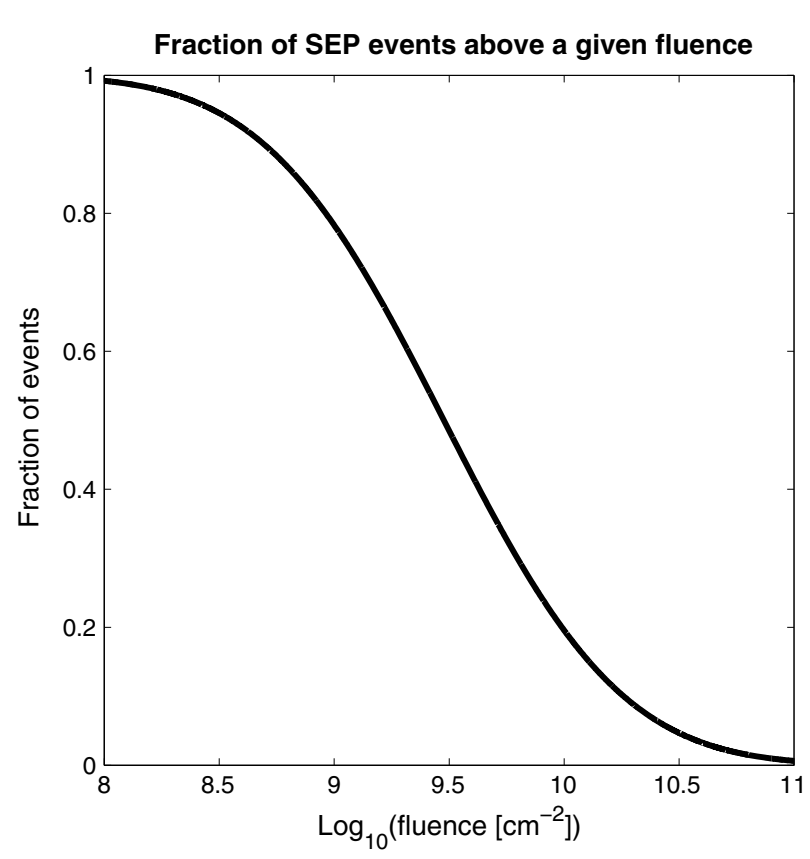

Figure 4. Fraction of SEP events with greater than a given fluence. or

$$
R(\geq F)=\frac{1}{2} R_{0} \operatorname{erfc}\left(\frac{F-\mu}{\sqrt{2} \sigma}\right)
$$

This rate should be regarded as a lower limit for SEP events capable of causing breakdown. Since even lower energy SEPs can induce breakdown and since they also typically have higher fluxes during events, the breakdown-inducing event rate should be greater than $R(\geq F)$. In other words, events not meeting the fluence criterion for $>1 \mathrm{MeV}$ protons may meet it at lower energies, depending on the energy spectra. With the JPL fluence model, however, we are unable to estimate this higher event rate, so we use $R(\geq F)$.

Finding the rate of events with a given fluence is only half of the solution, as described above. If the

fluence occurs over too long a time, i.e., if the average flux is too low, then the regolith can discharge without breakdown. In an SEP study based on the JPL fluence model, Jun et al. [2007] used >11.1 MeV proton data from IMP 8. They set an SEP event threshold of $>1 \mathrm{~cm}^{-2} \mathrm{~s}^{-1} \mathrm{sr}^{-1}$ averaged over a day (the same threshold used for the JPL fluence model for $>10 \mathrm{MeV}$ protons). They found 135 events during 14 active years, which corresponds to $\sim 9.6$ events per active year, while Feynman et al. [1993] found only 6.75 events per active year for $>10 \mathrm{MeV}$ protons. This disagreement occurs because Jun et al. [2007] defined an event's end as when the daily averaged flux fell below the threshold, whereas Feynman et al. [1993] required two days below threshold. Therefore, despite having a similar energy threshold, Jun et al. [2007] were able to distinguish more events.

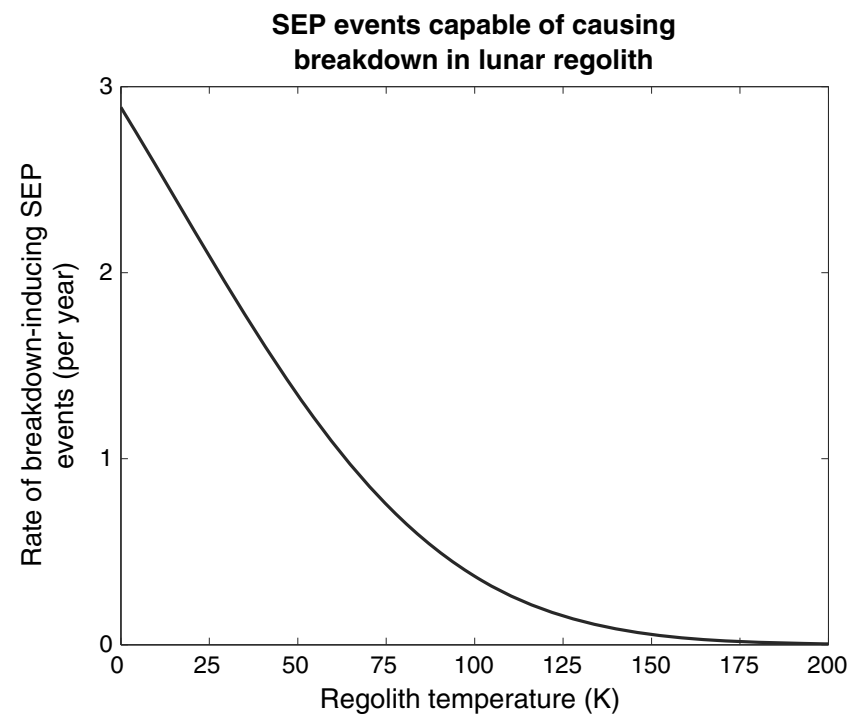

Figure 5. Estimated yearly rate of breakdown-inducing SEP events as a function of regolith temperature.
By fitting the event durations with an exponential, Jun et al. [2007] found that SEP events have a characteristic duration $\Delta t_{\text {Jun }}=5$ days. Since, however, the JPL proton fluence model defines events differently, it must have a correspondingly longer event duration $\Delta t$. Correctly estimating the event-averaged fluxes requires converting the duration found by Jun et al. [2007] to the longer one corresponding to the JPL model.

To do this, we note that the time spent above the event threshold summed over all events should be similar for the analyses of Jun et al. [2007] and Feynman et al. [1993], since they both used similar energy and flux thresholds. The only difference should be the 

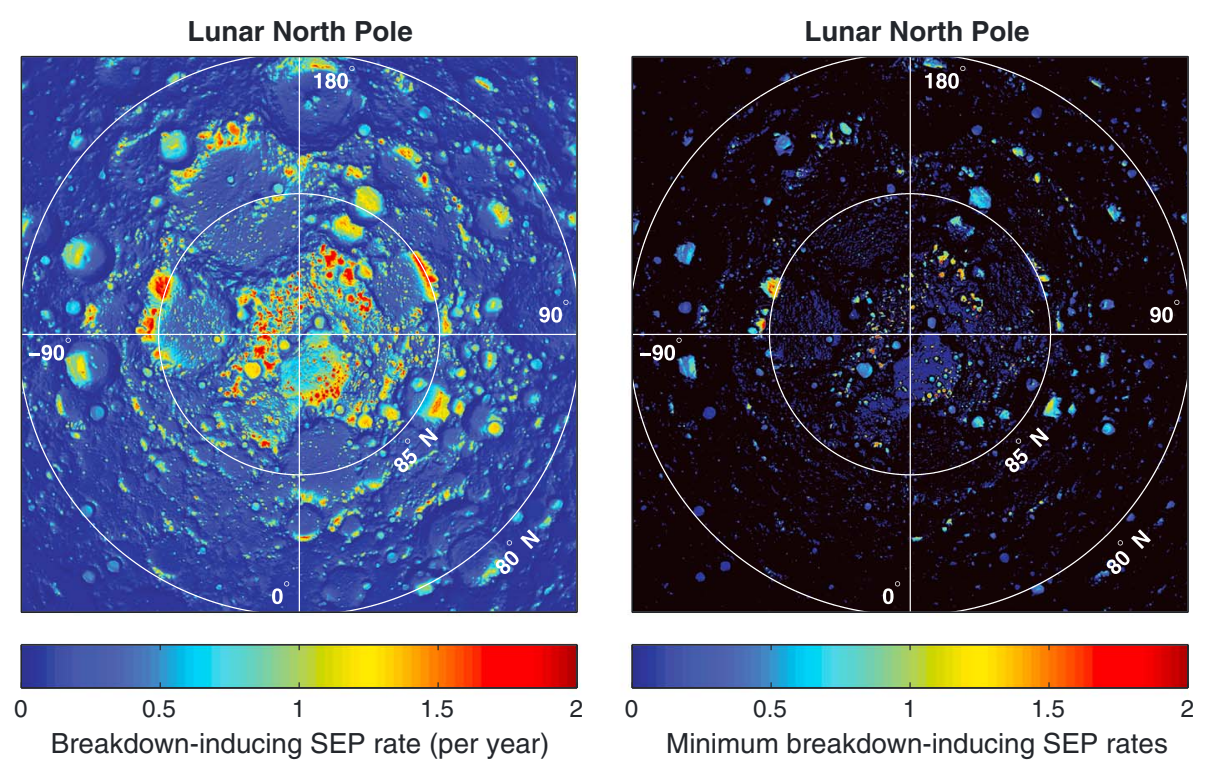

(per year)

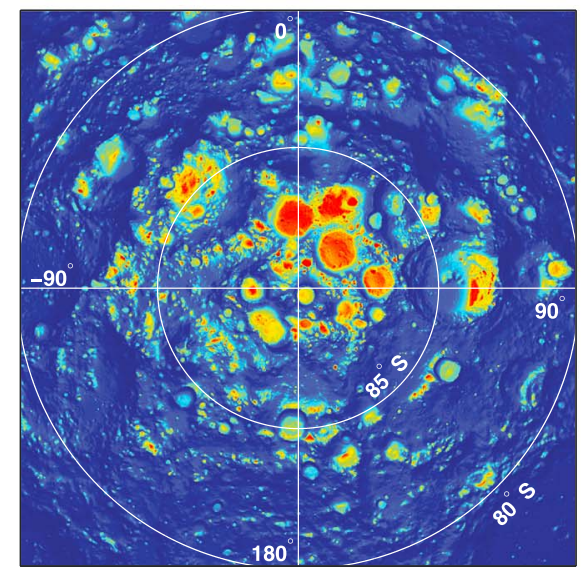

Lunar South Pole

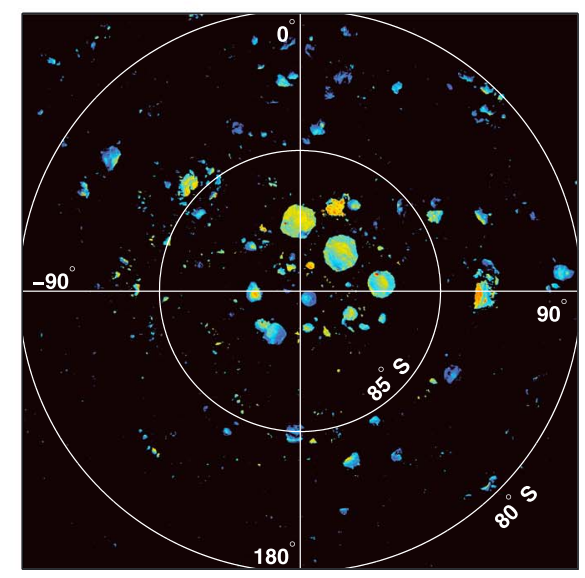

Lunar South Pole

Figure 6. Estimated yearly rate of breakdown-inducing SEP events for regolith at both poles. (left column) Based on average temperature at $2 \mathrm{~cm}$ depth and (right column) derived assuming regolith remains at the annual maximum surface temperature. Black-colored regions are where the maximum temperature $>200 \mathrm{~K}$.

characteristic event duration. Therefore, since $\Delta t_{\text {Jun }}$ and $\Delta t$ are the respective characteristic event durations of Jun et al. [2007] and Feynman et al. [1993], then

$$
\left(\frac{9.6 \text { events }}{\text { active } \mathrm{yr}}\right) \Delta t_{\text {Jun }} \approx\left(\frac{6.75 \text { events }}{\text { active } \mathrm{yr}}\right) \Delta t
$$

where their definition of active year is mentioned above (this equation is only approximate because Feynman et al. [1993] waited an extra day to end events and also used a slightly different integral energy channel). Since $\Delta t_{\text {Jun }}=5$ days, it must be that $\Delta t \approx 7$ days.

The average flux $\Phi$ throughout an event with fluence $f_{p}$ and lasting $\Delta t$ is thus

$$
\Phi=\frac{f_{p}}{\Delta t}
$$

(Note that, for a typical SEP event, which lasts for no more than a few days, most of the fluence occurs in less time than the event duration. Our method of determining the typical event duration assumes, however, that 


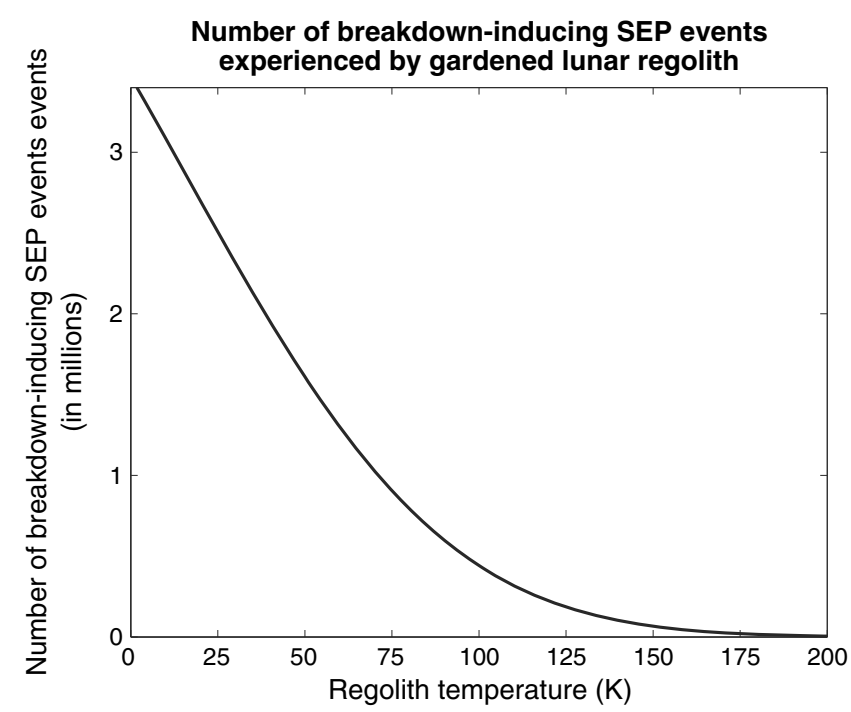

Figure 7. Estimated total number of breakdown-inducing SEP events in the gardened regolith as a function of its temperature. the flux stays constant throughout the event. Therefore, our method likely underestimates the characteristic flux.) The average flux needed for breakdown is

$$
\Phi_{B}=\frac{f_{B}}{\tau}
$$

where $f_{B}=10^{10} \mathrm{~cm}^{-2}$ is the fluence needed for breakdown in a solid [Violet and Frederickson, 1993; Frederickson et al., 1992]. Therefore, the criterion for breakdown is $\Phi \geq \Phi_{B}$ or

$$
\frac{f_{P}}{\Delta t} \geq \frac{f_{B}}{\tau}
$$

In other words, both the event's fluence $f_{p}$ and average flux $f_{p} / \Delta t$ must be great enough to cause breakdown. Solving for the event fluence and combining the result with equation (3) gives

$$
F \geq \log \left(\frac{\Delta t}{\tau} f_{B}\right)
$$

This means that the function $n\left(\geq \log \left[\frac{\Delta t}{\tau} f_{B}\right]\right)$ in equation (9) is the fraction of SEP events capable of causing breakdown for regolith with a discharging timescale $\tau$. Equations (1), (2), (11), and (16) all combine to give, as a function of regolith temperature, the rate of these SEP events:

$$
R(T)=\frac{1}{2} R_{0} \operatorname{erfc}\left(\frac{1}{\sqrt{2} \sigma}\left[\log _{10}\left(f_{B} \Delta t \epsilon^{-1} \sigma_{c 0} e^{\alpha T}\right)-\mu\right]\right)
$$

Again, because of the higher fluxes at lower energies, which are not accounted for here, we expect this rate to be a lower limit on the rate of SEP events capable of causing breakdown.

Figure 5 shows the rate of breakdown-inducing SEP events as a function of surface temperature, as derived in equation 17. Note that for events occurring once every $\geq 35$ years (a rate of $\sim 0.03$ per year), the length of the study of Feynman et al. [2002] makes this estimate unreliable, affecting estimates for regolith temperatures $\gtrsim 160 \mathrm{~K}$.

The coldest polar locations are predicted to experience SEP events capable of causing breakdown more than once per year, on average. For example, the impact site of LCROSS in Cabeus crater was at $40 \mathrm{~K}$ [Paige et al., 2010], corresponding to 1.6 event/yr. The coldest (29 K) location known on the Moon [Paige et al., 2010] would, on average, experience breakdown-inducing events about twice a year. We again use the model results of Paige et al. [2010], this time to map event rates at both poles in Figure 6. The coldest PSRs experience an average breakdown-inducing event rate nearly an order of magnitude greater than non-PSRs. Even assuming that the regolith remains at its maximum temperature, locations within many PSRs would still experience rates of $0.5-1$ event/yr.

To be able to understand the importance of this rate of breakdown-inducing SEP events, it is first necessary to estimate the total number of events the polar regolith has experienced. This, in turn, requires considering how meteoritic gardening affects this number. Because impacts on the Moon bury more surface area than they excavate, gardening is, on average, a burial process [Arnold, 1979]. Therefore, the meteoritic gardening rate limits the time a given layer can be subject to breakdown. On average, then, regolith will only be exposed to SEPs until it is buried by additional regolith to a depth of $\approx 1 \mathrm{~mm}$ - the penetration depth of the SEP electrons that are also present [Jordan et al., 2014]. This amount of burial requires roughly $1.2 \mathrm{Myr}$ [Arnold, 1975]. Gardening also thoroughly mixes this regolith, so we expect all the regolith throughout the gardened layer to have experienced breakdown-inducing events for $\approx 1.2 \mathrm{Myr}$, on average. (Note that this exposure time is independent of the gardening time. Note, too, that while vertical mixing has caused all 

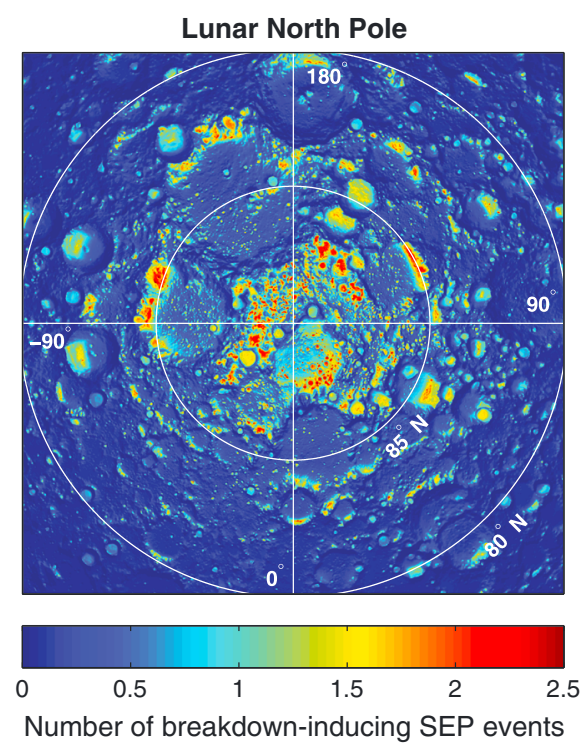

(in millions)

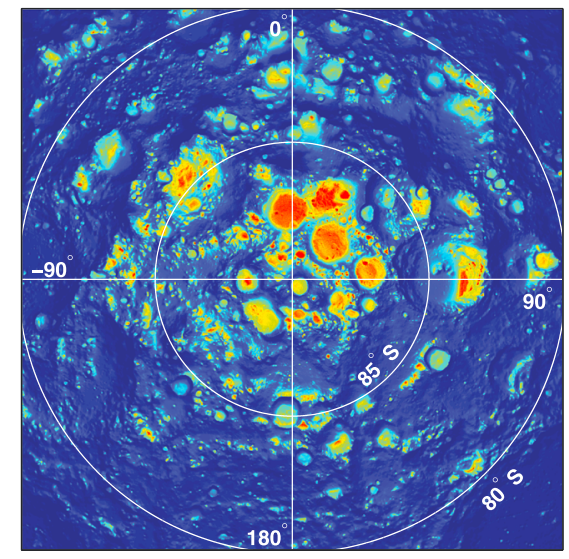

Lunar South Pole
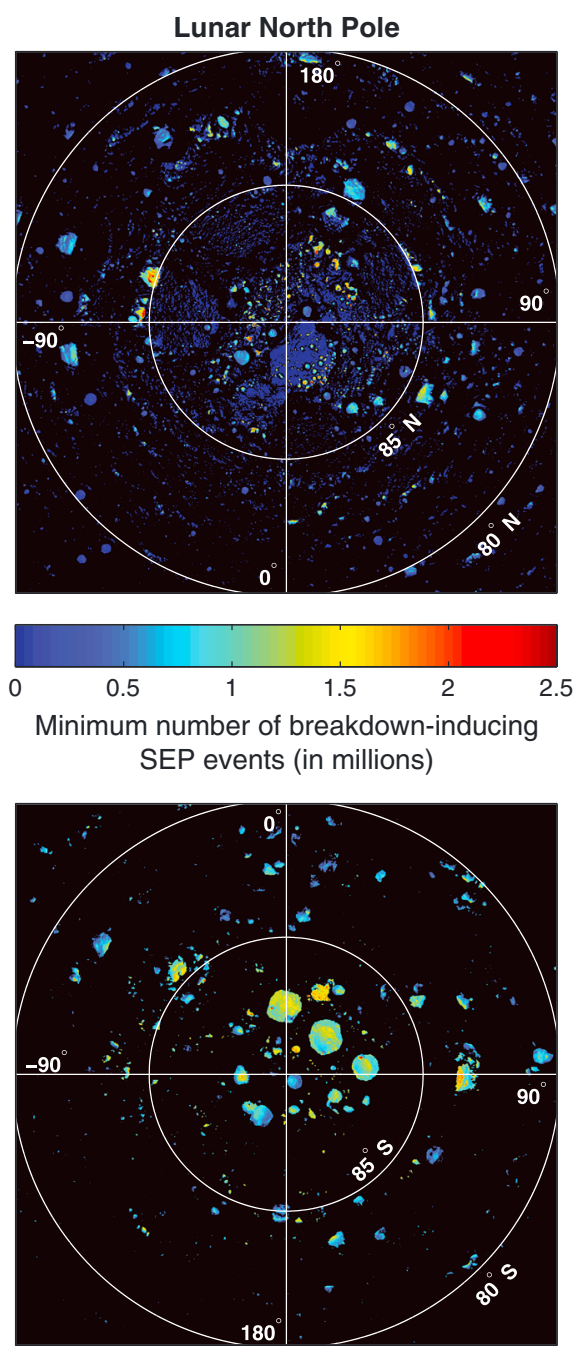

Lunar South Pole

Figure 8. Estimated total number of breakdown-inducing SEP events in the gardened regolith at both poles. (left column) Based on average temperature at $2 \mathrm{~cm}$ depth and (right column) derived assuming regolith remains at the annual maximum surface temperature. Black-colored regions are where the maximum temperature $>200 \mathrm{~K}$.

grains in the gardened zone at a given location to have approximately the same exposure time, their exposures have occurred at different times. For a more complete discussion see Jordan et al. [2013].) Therefore, regolith in the Moon's coldest regions is estimated to experience SEP events capable of causing breakdown about $2 \times 10^{6}$ times.

Figure 7 shows how the number of breakdown-causing events depends on temperature (as mentioned above, the number of events is unreliable for temperatures $\gtrsim 160 \mathrm{~K}$ ). Figure 8 shows maps of the total number of events estimated to have occurred at both poles, assuming 1.2 Myr of gardening. The PSRs have experienced at least 2 orders of magnitude more breakdown-inducing events than the warmest regions on the map. Again, even assuming an unrealistically high temperature (Figure 8, right column), the permanently shadowed regions have experienced only a factor of about 2 fewer breakdown-inducing SEP events.

Figure 9 shows how the number of breakdown-inducing SEP events experienced by the gardened regolith varies as a function of latitude (assuming both poles remain at the average temperatures shown in Figure 2). They both have experienced similar numbers of breakdown-inducing SEP events, peaking near the poles at nearly $10^{6}$ events/yr. Poleward of $\pm 80^{\circ}$, using the maximum temperature, instead of the average 


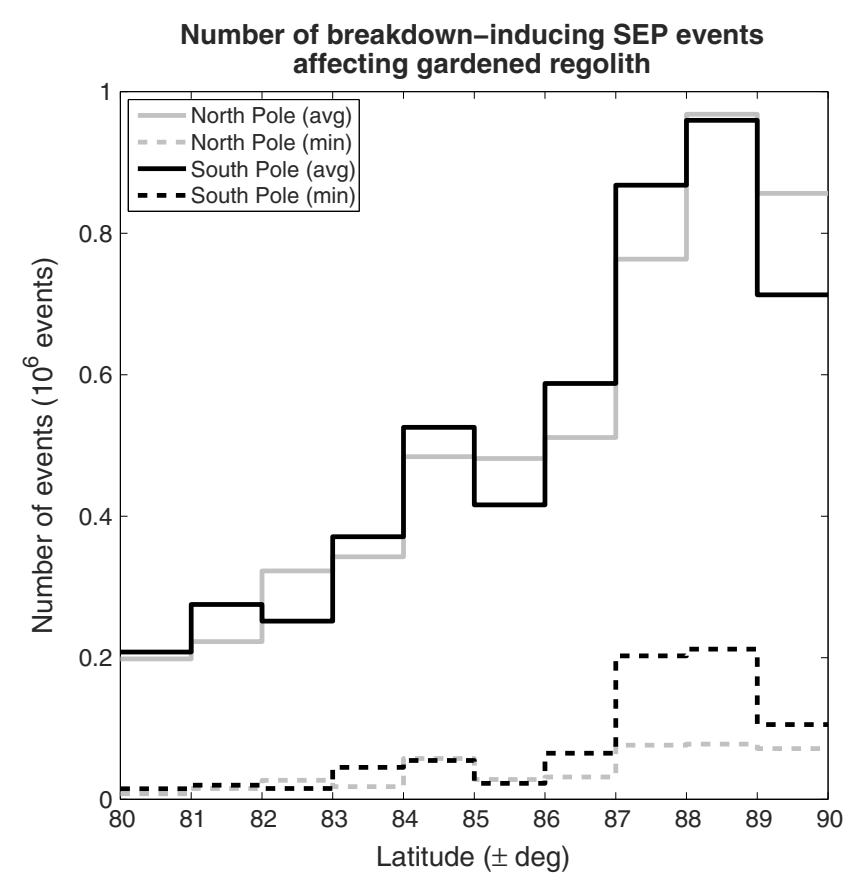

Figure 9. Estimated average number of breakdown-inducing SEP events in the gardened regolith in each $1^{\circ}$ latitude bin. Gray lines show rates near the north pole based on average temperature at $2 \mathrm{~cm}$ depth (solid) and on maximum surface temperature (dashed). Black lines show rates near the south pole.

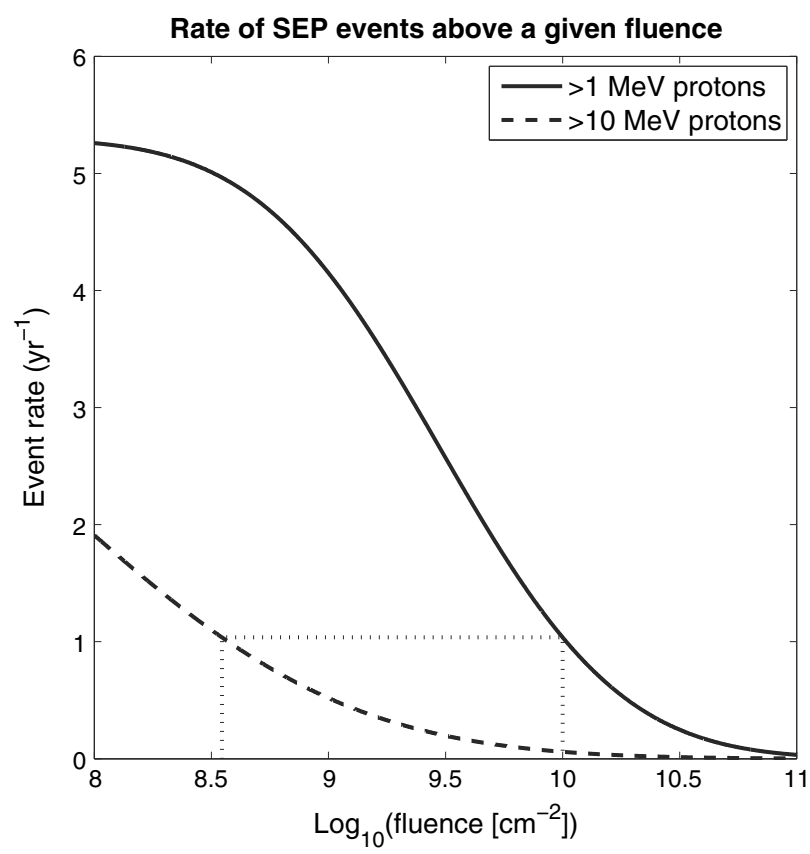

Figure 10. JPL fluence model's SEP event rates for $>1 \mathrm{MeV}$ and $>10 \mathrm{MeV}$ proton fluences [Feynman et al., 1993]. The dotted lines indicate both energy channels' fluences that correspond to the rate of $>1 \mathrm{MeV}$ breakdown-inducing events. temperature, decreases the rate of breakdown-inducing events by about an order of magnitude. This difference is most significant in the northern hemisphere. Near the south pole (poleward of $-87^{\circ}$ ), however, the change is less because of presence of relatively large permanently shadowed craters.

\section{Using CRaTER}

Before considering the effects of breakdown weathering, we outline a method to identify recent SEP events possibly capable of causing breakdown using LRO's Cosmic Ray Telescope for the Effects of Radiation (CRaTER). The telescope comprises three pairs of detectors separated by tissue-equivalent plastic [Spence et al., 2010]. Detector D1 is on the zenith end of the telescope during nominal orientation. An aluminum end cap shields D1 from protons $\lesssim 10 \mathrm{MeV}$, so $D 1$ count rates are mainly a measure of $>10 \mathrm{MeV}$ proton flux. D1's geometric factor is roughly $30 \mathrm{~cm}^{2} \mathrm{sr}$. Its field of view covers about half the sky, since the Moon blocks the other half (the flux of secondary particles from the Moon's surface is negligible [Wilson et al., 2012]).

While our work above has focused on $>1 \mathrm{MeV}$ protons, we can use $>10 \mathrm{MeV}$ protons as a proxy for the less energetic but more numerous protons. The JPL fluence model includes parameters describing the distribution of SEP event fluences for $>10 \mathrm{MeV}$ protons $\left(\mu=\log _{10}\left(7.3 \times 10^{7}\right)\right.$ and $\sigma=0.97$ ). The modeled SEP occurrence rates for both integral channels as a function of fluence are shown in Figure 10.

We want to find the $>10 \mathrm{MeV}$ proton fluence statistically associated with events whose $>1 \mathrm{MeV}$ protons can cause breakdown. In other words, we are looking for the $>10 \mathrm{MeV}$ proton fluence that occurs at the same rate as the breakdown fluence for $>1 \mathrm{MeV}$ protons. Not every event meeting the $>1 \mathrm{MeV}$ criterion will also meet the $>10 \mathrm{MeV}$ criterion; that depends 


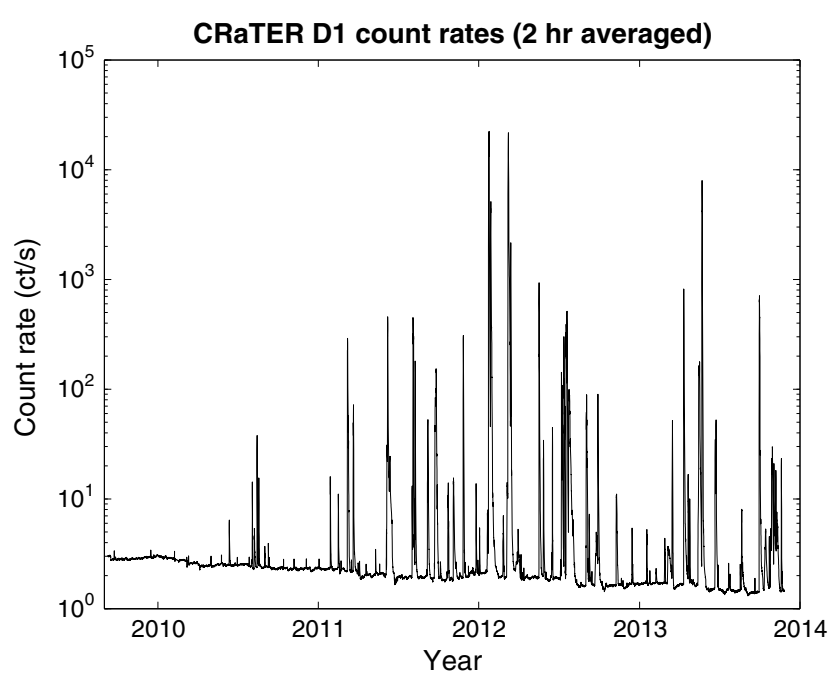

Figure 11. Two hour averaged, $>10 \mathrm{MeV}$ proton count rates from CRaTER D1. on the hardness of the event's energy spectrum. We are simply looking for a statistical way to use the $>10 \mathrm{MeV}$ protons detected by CRaTER to infer when a potentially breakdown-inducing event might occur at the Moon. The rate of such events is $\approx 1$ per year. As shown in the figure, we find that a $>10 \mathrm{MeV}$ fluence of $\approx 3 \times 10^{8} \mathrm{~cm}^{-2}$ statistically occurs with the same frequency as a $>1 \mathrm{MeV}$ fluence of $\approx 10^{10} \mathrm{~cm}^{-2}$.

In Figure 11, we show $2 \mathrm{~h}$ averaged D1 count rates. Many SEP events are visible in the plot. (The $2 \mathrm{~h}$ averaging removes the slight variation in count rates due to LRO's orbit. For the first part of the mission-from 9 September 2009 to 11 December 2011 - the spacecraft was in a circular orbit $\sim 50 \mathrm{~km}$ above the lunar surface. Since then, LRO has been in a more elliptical "parking" orbit with a $2 \mathrm{~h}$ period. Although this means that, on average, the spacecraft is farther from the Moon than before, the effect on the overall count rate-only a few percent-is negligible for our purpose.)

To find breakdown-inducing events in the CRaTER data, we convert the count rates to fluences by multiplying them by the field of view (roughly $2 \pi \mathrm{sr}$, since the Moon blocks about half the sky) and dividing by the detector's geometric factor. As shown in section 3, the typical SEP event length in this study was $\approx 7$ days. We therefore show the 7 day sliding fluence for D1 in Figure 12. The horizontal dashed line indicates the $3 \times 10^{8} \mathrm{~cm}^{-2}$ threshold. Two events (starting 23 January 2012 and 7 March 2012) during the LRO mission surpassed this threshold and therefore may have caused dielectric breakdown in the Moon's polar regions. CRaTER's continuing operation will enable us to identify new events as they occur and perhaps correlate them with observations from other instruments on LRO.

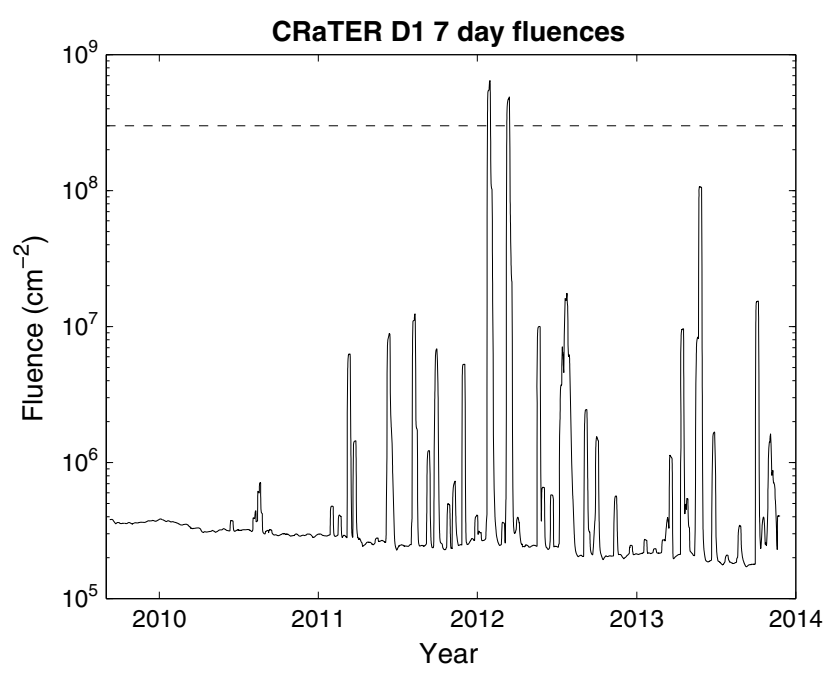

Figure 12. CRaTER D1 7 day fluences of $>10 \mathrm{MeV}$ protons. The dashed line indicates the breakdown threshold.

\section{Effects of Breakdown Weathering}

Breakdown weathering has potentially three main effects. First, along with meteorites, it could help drive comminution (the fragmentation of the regolith). Second, it may increase regolith porosity, and third, it could lower the regolith's albedo. We consider each in turn.

Comminution, as normally conceived, is a process by which meteoroid impacts fracture the regolith into smaller grains. The finest fragments tend to be single minerals [Devine et al., 1982; Horz et al., 1984]. Both full and partial breakdown can similarly weather the regolith, because, as discussed in section 2 , 


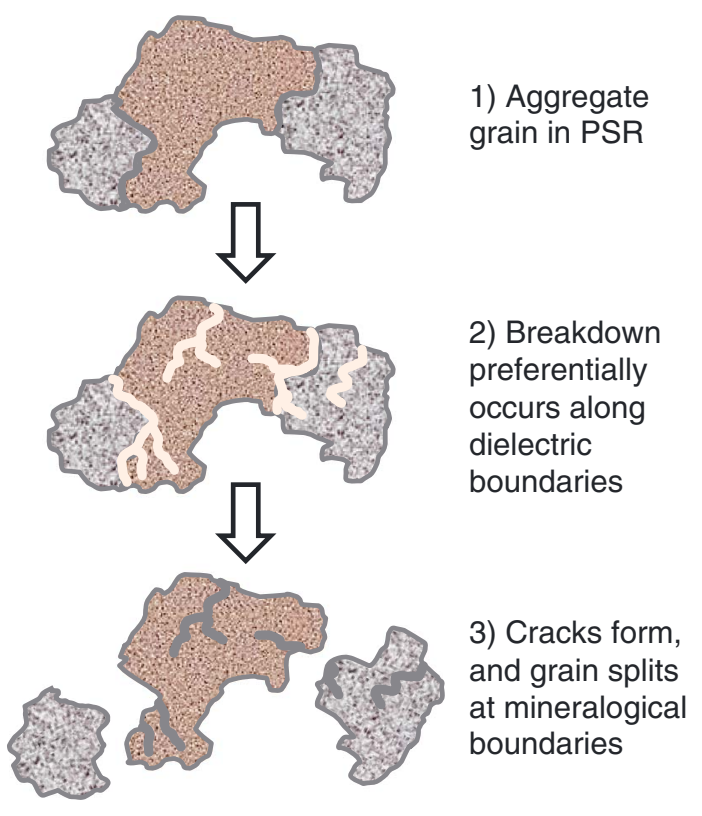

Figure 13. Cartoon showing how dielectric breakdown could fragment lunar regolith grains. Cracks formed are more likely to experience repeated breakdown. it tends to create cracks along mineralogical boundaries [Andres et al., 2001b], thus weakening the grain or possibly fragmenting it (see Figure 13). These cracks also increase the chance that repeated breakdown will occur at those locations [Lisitsyn et al., 1998]. Even if breakdown itself has not fragmented a grain, it is conceivable that any ensuing meteoroid impact is more likely to split it at the weakened location. Therefore, since PSRs experience more breakdown than other areas on the Moon, we would expect them to have finer grains and a higher percentage of monomineralic grains.

Second, by splitting grains, breakdown weathering can increase the porosity of the regolith within PSRs. (Note that the porosity also slightly increases as breakdown channels form.) If breakdown splits a small grain, the expanding plasma may cause the resulting fragments to move

a small distance [Adamo and Nanevicz, 1975; Campins and Krider, 1989]. In this way, breakdown weathering may help split regolith particles into fragments small enough to be dominated by van der Waals forces and may also help give them the initial motion needed to form fine, porous structures (i.e., "fairy castles") that will not collapse under gravity [Hapke and van Horn, 1963]. Schultz et al. [2010] pointed out that the lack of an obvious flash during the LCROSS impact could indicate a $>70 \%$ regolith porosity, and Gladstone et al. [2012], using LRO's Lyman Alpha Mapping Project (LAMP) far-ultraviolet albedo observations, found that a similar porosity may be characteristic of many PSRs (compared with $40 \%$ porosity of regolith outside PSRs). Breakdown weathering may contribute to the formation of these putative fairy castle structures in the upper $(\approx 1 \mathrm{~mm})$ layer of regolith (see Figure 14).

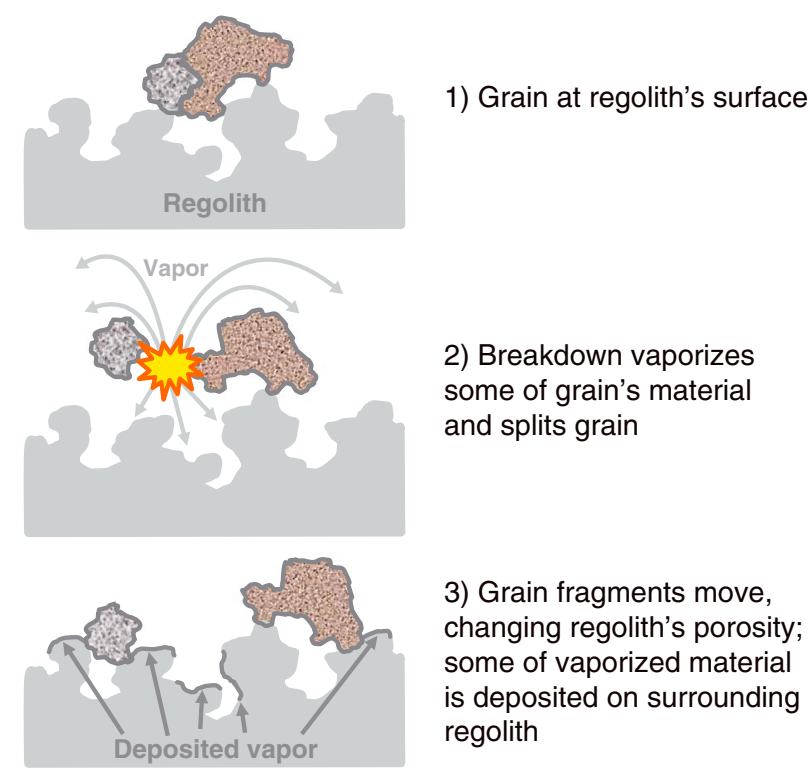

Figure 14. Cartoon showing how dielectric breakdown could change lunar regolith's porosity and albedo by splitting grains and vaporizing some of the grain's material.
Third, breakdown weathering can affect albedo in two ways. First, the previously mentioned increased porosity-channels/cracks and fairy castles-reduces the albedo, because light incident on a porous structure is more likely to be absorbed [Hapke and van Horn, 1963]. Second, since breakdown vaporizes the material, the ejected vapor can condense on surrounding grains (see Figure 14). After many breakdown events, the regolith's albedo could decrease, since such vapor deposition likely lowers albedo [Hapke, 1973; Hapke et al., 1975].

These effects of breakdown weathering could explain the LAMP albedo observations. As Gladstone et al. [2012] pointed out, the Lyman $\alpha$ albedo correlates with Diviner temperature data (lower albedo in colder regions) within craters that are only partly in permanent 
shadow. Because breakdown weathering is temperature dependent, it may explain this correlation. In other words, colder regions are more likely to experience breakdown and will thus have lower albedo. Breakdown weathering may therefore contribute significantly to the decreased albedo observed in the polar regions.

This albedo change could be tested in experiments that cause breakdown by exposing regolith samples or simulants to energetic charged particles.

It is possible that these breakdown events could be observed remotely. Breakdown in solids creates ultraviolet line spectra [Budenstein et al., 1969] and emission in at least the 1-10 MHz range [Green and Frederickson, 2006]. In principle, these emissions should be detectable, and we are attempting to determine whether they may have already been detected, either by LRO or ground-based instruments.

\section{Conclusions}

We have shown that breakdown weathering could be an important process in the Moon's polar regions. We have used LRO/CRaTER particle data to show that two breakdown-inducing events may have occurred since LRO arrived at the Moon. We have also shown that all gardened regolith within PSRs has likely experienced up to $2 \times 10^{6}$ SEP events capable of causing breakdown, while the warmest polar regions have experienced about 2 orders of magnitude fewer events. These estimates, however, are probably too low for two reasons. First, partial breakdown aging can still occur with weaker electric fields (i.e., lower energetic particle fluxes), thus increasing the regolith's susceptibility to breakdown. Second, the rate of potentially breakdown-causing SEP events is likely higher than we have estimated, because we have only considered $>1 \mathrm{MeV}$ protons, whereas smaller SEP events may still have sufficient fluxes and fluences of lower energy particles to cause breakdown.

Breakdown weathering, therefore, possibly plays an important role in the physical properties of the polar regolith. If it occurs, it is expected to increase the percentage of fine and monomineralic grains. This may also cause the formation of fairy castle structures and contribute to the low-albedo features detected by LRO/LAMP, possibly establishing a correlation between these features and the average surface temperatures within craters that are only partly in permanent shadow.

\section{Acknowledgments}

This work was supported by NASA grants NNG11PA03C, NNX10AB17A, and NNX14AG13A. The authors wish to thank Alex Boyd for helpful discussions. The authors also thank the ACE/EPAM team and its Principa Investigator Robert Gold of JHU/APL for the providing the ACE data via CDAWeb at http://cdaweb.gsfc.nasa. gov/. The LRO/Diviner Level 4 Polar Resource Products and LRO/CRaTER Level 2 data are available at the NASA Planetary Data System at http://pds. nasa.gov. The authors also thank the two reviewers for their helpful comments and suggestions.

\section{References}

Adamo, R. C., and J. E. Nanevicz (1975), Spacecraft-charging studies of voltage breakdown processes on spacecraft thermal control mirrors, in Spacecraft Charging by Magnetospheric Plasmas, edited by R. C. Adamo, and J. E. Nanevicz, pp. 225-235, Am. Inst. of Aeronat. and Astronaut., Princeton, N. J.

Andres, U., I. Timoshkin, J. Jirestig, and H. Stallknecht (2001a), Liberation of valuable inclusions in ores and slags by electrical pulses, Powder Technol., 114(1), 40-50.

Andres, U., I. Timoshkin, and M. Soloviev (2001b), Energy consumption and liberation of minerals in explosive electrical breakdown of ores, Miner. Process. Extractive Metall., 110(3), 149-157, doi:10.1179/mpm.2001.110.3.149.

Arnold, J. R. (1975), Monte Carlo simulation of turnover processes in the lunar regolith, in Lunar Science Conference, vol. 6, pp. 2375-2395, Pergamon Press, New York.

Arnold, J. R. (1979), Ice in the lunar polar regions, J. Geophys. Res., 84, 5659-5668, doi:10.1029/JB084iB10p05659.

Bahder, G., T. Garrity, M. Sosnowski, R. Eaton, and C. Katz (1982), Physical model of electric aging and breakdown of extruded polymeric insulated power cables, IEEE Trans. Power Apparatus Syst., PAS-101(6), 1379-1390.

Budenstein, P. P. (1980), On the mechanism of dielectric breakdown of solids, IEEE Trans. Electr. Insul., El-15(3), $225-240$.

Budenstein, P. P., P. J. Hayes, J. L. Smith, and W. B. Smith (1969), Destructive breakdown in thin films of $\mathrm{SiO}_{1} \mathrm{MgF}_{2}, \mathrm{CaF}_{2}, \mathrm{CeF}_{3}, \mathrm{CeO}_{2}$ and Teflon, J. Vac. Sci. Technol., 6(2), 289-303, doi:10.1116/1.1492680.

Buhler, C., C. Calle, J. Clements, J. Mantovani, and M. Ritz (2007), Test method for in situ electrostatic characterization of lunar dust, in IEEE Aerospace Conference, pp. 1-19, IEEE, Big Sky, Mont., doi:10.1109/AERO.2007.352755.

Campins, H., and E. P. Krider (1989), Surface discharges on natural dielectrics in the solar system, Science, 245, 622-624, doi:10.1126/science.245.4918.622.

Cane, H. V., R. E. McGuire, and T. T. von Rosenvinge (1986), Two classes of solar energetic particle events associated with impulsive and long-duration soft X-ray flares, Astrophys. J., 301, 448-459, doi:10.1086/163913.

Colaprete, A. et al. (2010), Detection of Water in the LCROSS Ejecta Plume, Science, 330, 463-468, doi:10.1126/science.1186986.

Devine, J. M., D. S. McKay, and J. J. Papike (1982), Lunar regolith-Petrology of the less than 10 micron fraction, in Lunar and Planetary Science Conference Proceedings, vol. 13, edited by J. M. Devine, D. S. McKay, and J. J. Papike, pp. A260-A268, AGU, Washington, D. C.

Evenson, P., P. Meyer, S. Yanagita, and D. J. Forrest (1984), Electron-rich particle events and the production of gamma-rays by solar flares, Astrophys. J., 283, 439-449, doi:10.1086/162323.

Farrell, W. M., T. J. Stubbs, J. S. Halekas, R. M. Killen, G. T. Delory, M. R. Collier, and R. R. Vondrak (2010), Anticipated electrical environment within permanently shadowed lunar craters, J. Geophys. Res., 115, E03004, doi:10.1029/2009JE003464.

Feynman, J., T. P. Armstrong, L. Dao-Gibner, and S. Silverman (1990), New interplanetary proton fluence model, J. Spacecr. Rockets, 27, 403-410, doi:10.2514/3.26157.

Feynman, J., G. Spitale, J. Wang, and S. Gabriel (1993), Interplanetary proton fluence model-JPL 1991, J. Geophys. Res., 98, 13,281-13,294, doi:10.1029/92JA02670.

Feynman, J., A. Ruzmaikin, and V. Berdichevsky (2002), The JPL proton fluence model: An update, J. Atmos. Sol. Terr. Phys., 64, 1679-1686, doi:10.1016/S1364-6826(02)00118-9. 
Frederickson, A. R., D. B. Cotts, J. A. Wall, and F. L. Bouquet (1986), Spacecraft Dielectric Material Properties and Spacecraft Charging, Am. Inst. of Aeronaut. and Astronaut., Univ. of Michigan.

Frederickson, A. R., E. G. Holeman, and E. G. Mullen (1992), Characteristics of spontaneous electrical discharging of various insulators in space radiations, IEEE Trans. Nucl. Sci., 39, 1773-1782, doi:10.1109/23.211366.

Fujita, T., I. Yoshimi, A. Shibayama, T. Miyazaki, K. Abe, M. Sato, W. T. Yen, and J. Svoboda (2001), Crushing and liberation of materials by electrical disintegration, Eur. J. Mineral Process. Environ. Protect., 1(2), 113-122.

Funkhouser, J., E. Jessberger, O. Müller, and J. Zähringer (1971), Active and inert gases in Apollo 12 and Apollo 11 samples released by crushing at room temperature and by heating at low temperatures, in Lunar and Planetary Science Conference Proceedings, vol. 2, pp. 1381-1396, Lunar and Planet. Inst., Stony Brook, N. Y.

Garrett, H. B., and R. W. Evans (2001), Internal electrostatic discharge environment at Jupiter, in Proceedings of the Seventh International Conference Spacecraft Charging Technology, vol. 476, edited by R. A. Harris, pp. 609-616, ESA Spec. Publ., Noordwijk, Netherlands.

Gladstone, G. R., et al. (2012), Far-ultraviolet reflectance properties of the Moon's permanently shadowed regions, J. Geophys. Res. Planet, 117, EOOH04, doi:10.1029/2011JE003913.

Gosling, J. T. (1993), The solar flare myth, J. Geophys. Res., 98, 18,937-18,950, doi:10.1029/93JA01896.

Green, N. W., and J. R. Dennison (2008), Deep dielectric charging of spacecraft polymers by energetic protons, IEEE Trans. Plasma Sci., 36, 2482-2490, doi:10.1109/TPS.2008.2003442.

Green, N. W., and A. R. Frederickson (2006), A study of spacecraft charging due to exposure to interplanetary protons, in Space Technology and Applications International Forum - STAIF, vol. 813, edited by M. S. El-Genk, pp. 694-700, American Institute of Physics Conference Series, Jet Propulsion Laboratory, National Aeronautics and Space Administration, Pasadena, Calif., doi:10.1063/1.2169250.

Halekas, J. S., et al. (2007), Extreme lunar surface charging during solar energetic particle events, Geophys. Res. Lett., 34, L02111, doi:10.1029/2006GL028517.

Halekas, J. S., G. T. Delory, R. P. Lin, T. J. Stubbs, and W. M. Farrell (2008), Lunar Prospector observations of the electrostatic potential of the lunar surface and its response to incident currents, J. Geophys. Res., 113, A09102, doi:10.1029/2008JA013194.

Halekas, J. S., G. T. Delory, R. P. Lin, T. J. Stubbs, and W. M. Farrell (2009), Lunar surface charging during solar energetic particle events: Measurement and prediction, J. Geophys. Res., 114, A05110, doi:10.1029/2009JA014113.

Hapke, B. (1973), Darkening of silicate rock powders by solar wind sputtering, Moon, 7, 342-355, doi:10.1007/BF00564639.

Hapke, B., and H. van Horn (1963), Photometric studies of complex surfaces, with applications to the Moon, J. Geophys. Res., 68, $4545-4570$.

Hapke, B., W. Cassidy, and E. Wells (1975), Effects of vapor-phase deposition processes on the optical, chemical, and magnetic properties of the lunar regolith, Moon, 13, 339-353, doi:10.1007/BF00567525.

Hara, M., and H. Okubo (1998), Electrical insulation characteristics of superconducting power apparatus, Cryogenics, 38(11), 1083-1093, doi:10.1016/S0011-2275(98)00095-2.

Horz, F., M. J. Cintala, T. H. See, F. Cardenas, and T. D. Thompson (1984), Grain size evolution and fractionation trends in an experimental regolith, in Lunar and Planetary Science Conference Proceedings, vol. 15, pp. 183-196, Lunar and Planet. Inst., Houston, Tex.

Jordan, A. P., T. J. Stubbs, C. J. Joyce, N. A. Schwadron, H. E. Spence, and J. K. Wilson (2013), The formation of molecular hydrogen from water ice in the lunar regolith by energetic charged particles, J. Geophys. Res. Planets, 118, 1257-1264, doi:10.1002/jgre.20095.

Jordan, A. P., T. J. Stubbs, J. K. Wilson, N. A. Schwadron, H. E. Spence, and C. J. Joyce (2014), Deep dielectric charging of regolith within the Moon's permanently shadowed regions, J. Geophys. Res. Planet, 119, 1806-1821, doi:10.1002/2014JE004648.

Jun, I., R. T. Swimm, A. Ruzmaikin, J. Feynman, A. J. Tylka, and W. F. Dietrich (2007), Statistics of solar energetic particle events: Fluences, durations, and time intervals, Adv. Space Res., 40, 304-312, doi:10.1016/j.asr.2006.12.019.

Kirkici, H., M. F. Rose, and T. Chaloupka (1996), Experimental study on simulated lunar soil: High voltage breakdown and electrical insulation characteristics, IEEE Trans. Dielectr. Electr. Insul., 3(1), 119-125.

Lisitsyn, I. V., H. Inoue, I. Nishizawa, S. Katsuki, and H. Akiyama (1998), Breakdown and destruction of heterogeneous solid dielectrics by high voltage pulses, J. Appl. Phys., 84(11), 6262-6267.

McGuire, R. E., and T. T. von Rosenvinge (1984), The energy spectra of solar energetic particles, Adv. Space Res., 4, 117-125, doi:10.1016/0273-1177(84)90301-6.

McKay, D. S., G. Heiken, A. Basu, G. Blanford, S. Simon, R. Reedy, B. M. French, and J. Papike (1991), The lunar regolith, in Lunar Sourcebook, edited by G. Heiken, D. Vaniman, and B. M. French, pp. 285-356, Cambridge Univ., Cambridge.

Neish, C. D., D. B. J. Bussey, P. Spudis, W. Marshall, B. J. Thomson, G. W. Patterson, and L. M. Carter (2011), The nature of lunar volatiles as revealed by Mini-RF observations of the LCROSS impact site, J. Geophys. Res. Planet, 116, E01005, doi:10.1029/2010JE003647.

Olhoeft, G. R., and D. W. Strangway (1975), Dielectric properties of the first 100 meters of the moon, Earth Planet. Sci. Lett., 24, 394-404, doi:10.1016/0012-821X(75)90146-6.

Olhoeft, G. R., A. L. Frisillo, and D. W. Strangway (1974a), Electrical properties of lunar soil sample 15301,38, J. Geophys. Res., 79, 1599-1604, doi:10.1029/JB079i011 p01599.

Olhoeft, G. R., A. L. Frisillo, D. W. Strangway, and H. Sharpe (1974b), Temperature dependence of electrical conductivity and lunar temperatures, The Moon, 9, 79-87, doi:10.1007/BF00565394.

Paige, D. A., et al. (2010), Diviner lunar radiometer observations of cold traps in the Moon's south polar region, Science, 330, 479-482, doi:10.1126/science.1187726.

Petrenko, V. F. (1993), Electrical properties of ice, Spec. Rep. 93-20, US Army Cold Regions Research and Engineering Laboratory, Hanover, N. H.

Reames, D. V. (1999), Particle acceleration at the Sun and in the heliosphere, Space Sci. Rev., 90, 413-491, doi:10.1023/A:1005105831781.

Reedy, R. C. (1980), Lunar radionuclide records of average solar-cosmic-ray fluxes over the last ten million years, in The Ancient Sun: Fossil Record in the Earth, Moon and Meteorites, edited by R. O. Pepin, J. A. Eddy, and R. B. Merrill, pp. 365-386, Pergamon, New York.

Roedder, E., and P. W. Weiblen (1970), Lunar petrology of silicate melt inclusions, Apollo 11 rocks, Geochim. Cosmochim. Ac. Suppl., 1, pp. 801-837.

Russ, G. P., III, and M. T. Emerson III (1980), ${ }^{53} \mathrm{Mn}$ and ${ }^{26} \mathrm{Al}$ evidence for solar cosmic ray constancy-An improved model for interpretation, in The Ancient Sun: Fossil Record in the Earth, Moon and Meteorites, edited by R. O. Pepin, J. A. Eddy, and R. B. Merrill, pp. 387-399, Pergamon, New York.

Schultz, P. H., B. Hermalyn, A. Colaprete, K. Ennico, M. Shirley, and W. S. Marshall (2010), The LCROSS cratering experiment, Science, 330 , 468-472, doi:10.1126/science.1187454.

Smart, D. F., and M. A. Shea (1985), Galactic cosmic radiation and solar energetic particles, in Handbook of Geophysics and the Space Environment, edited by A. S. Jursa, pp. 6-1-6-29, U.S. Air Force Geophysics Laboratories, Hanscom Air Force Base, Mass. 
Sørensen, J., D. Rodgers, K. Ryden, P. Latham, G. Wrenn, L. Levy, and G. Panabiere (1999), Esa's tools for internal charging, in 5th European Conference on Radiation and Its Effects on Components and Systems (RADECS), pp. 27-33, IEEE, Fontevraud, France, doi:10.1109/RADECS.1999.858540.

Spence, H. E., et al. (2010), CRaTER: The cosmic ray telescope for the effects of radiation experiment on the lunar reconnaissance orbiter mission, Space Sci. Rev., 150, 243-284, doi:10.1007/s11214-009-9584-8.

Stubbs, T. J., W. M. Farrell, J. S. Halekas, J. K. Burchill, M. R. Collier, M. I. Zimmerman, R. R. Vondrak, G. T. Delory, and R. F. Pfaff (2014), Dependence of lunar surface charging on solar wind plasma conditions and solar irradiation, Planet. Space Sci., 90, 10-27, doi:10.1016/j.pss.2013.07.008.

Violet, M. D., and A. R. Frederickson (1993), Spacecraft anomalies on the CRRES satellite correlated with the environment and insulator samples, IEEE Trans. Nucl. Sci., 40, 1512-1520, doi:10.1109/23.273511.

Wilson, J. K., et al. (2012), The first cosmic ray albedo proton map of the Moon, J. Geophys. Res. Planet, 117, E0OH23, doi:10.1029/2011JE003921.

Zimmerman, M. I., W. M. Farrell, T. J. Stubbs, J. S. Halekas, and T. L. Jackson (2011), Solar wind access to lunar polar craters: Feedback between surface charging and plasma expansion, Geophys. Res. Lett., 38, L19202, doi:10.1029/2011GL048880.

Zimmerman, M. I., T. L. Jackson, W. M. Farrell, and T. J. Stubbs (2012), Plasma wake simulations and object charging in a shadowed lunar crater during a solar storm, J. Geophys. Res., 117, E00K03, doi:10.1029/2012JE004094. 